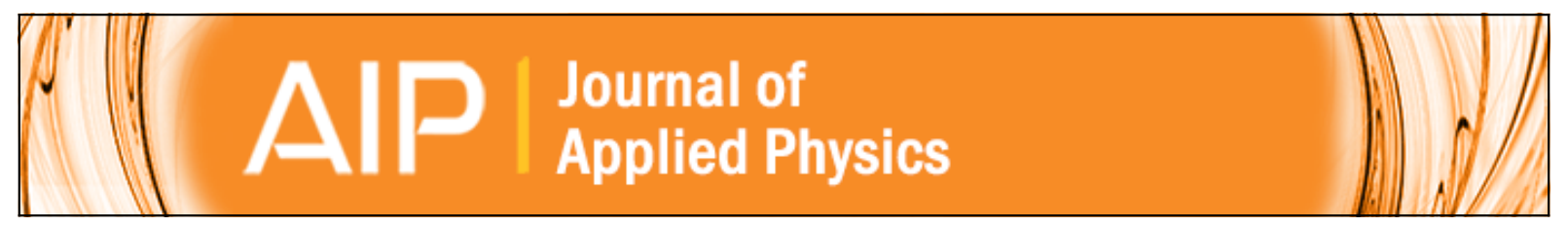

\title{
Onset of implant-related recombination in self-ion implanted and annealed crystalline
} silicon

Daniel Macdonald, Prakash N. K. Deenapanray, and Stephan Diez

Citation: Journal of Applied Physics 96, 3687 (2004); doi: 10.1063/1.1789630

View online: http://dx.doi.org/10.1063/1.1789630

View Table of Contents: http://scitation.aip.org/content/aip/journal/jap/96/7?ver=pdfcov

Published by the AIP Publishing

\section{Articles you may be interested in}

Carrier lifetime studies of deeply penetrating defects in self-ion implanted silicon

Appl. Phys. Lett. 82, 2987 (2003); 10.1063/1.1572469

High resolution electrical studies of vacancy-rich and interstitial-rich regions in ion-implanted silicon

J. Appl. Phys. 93, 5118 (2003); 10.1063/1.1564286

Boron segregation to extended defects induced by self-ion implantation into silicon

J. Appl. Phys. 85, 7597 (1999); 10.1063/1.370560

Evolution of interstitial- and vacancy-type defects upon thermal annealing in ion-implanted $\mathrm{Si}$

Appl. Phys. Lett. 71, 389 (1997); 10.1063/1.119546

Evolution from point to extended defects in ion implanted silicon

J. Appl. Phys. 82, 120 (1997); 10.1063/1.365583

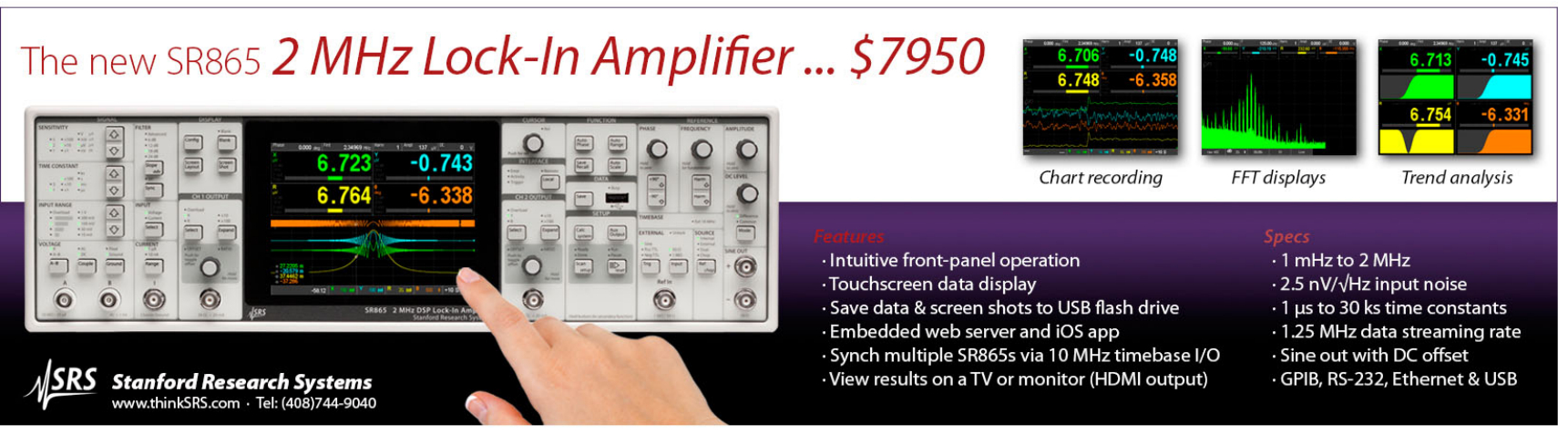




\title{
Onset of implant-related recombination in self-ion implanted and annealed crystalline silicon
}

\author{
Daniel Macdonald \\ Department of Engineering, Faculty of Engineering and Information Technology, The Australian National \\ University, Canberra, ACT 0200, Australia \\ Prakash N. K. Deenapanray \\ Center for Sustainable Energy Systems, Department of Engineering, Faculty of Engineering \\ and Information Technology, The Australian National University, Canberra, ACT 0200, Australia \\ Stephan Diez \\ Fraunhofer Institute for Solar Energy Systems ISE, Heidenhofstrasse 2, D-79110 Freiburg, Germany
}

(Received 10 May 2004; accepted 13 July 2004)

\begin{abstract}
The impact of residual recombination centers after low-energy self-implantation of crystalline silicon wafers and annealing at $900{ }^{\circ} \mathrm{C}$ has been determined by bulk carrier lifetime measurements as a function of implant dose. Doses below $10^{13} \mathrm{~cm}^{-2}$ resulted in no measurable increase in recombination, while higher doses caused a linear increase in the recombination center density. This threshold value corresponds to the known critical dose required for the formation of relatively stable dislocation loops during high temperature annealing. Deep level transient spectroscopy revealed a decrease in the vacancy-related defect concentration in the high-dose samples, which we interpret as reflecting an increase in the silicon interstitial concentration. This suggests that silicon interstitials, arising from the slowly dissolving dislocation loops, may be responsible for the increased recombination deep within the samples. (C) 2004 American Institute of Physics.
\end{abstract}

[DOI: $10.1063 / 1.1789630]$

\section{INTRODUCTION}

The recombination properties of very low concentrations of metallic impurities in silicon are of great interest in both microelectronics and photovoltaics. There are several approaches of preparing samples with such dilute impurities for characterization purposes. One possibility is to introduce contaminants during ingot growth. However, nonuniform profiles arising from low segregation coefficients, and precipitation due to low solubility limits, can result in uncertain outcomes. Similarly, dipping wafers into dilute solutions followed by annealing does not usually allow the final concentration in the wafer to be accurately estimated. A more certain approach is to use ion implantation, which allows very low and well-controlled concentrations to be introduced. Annealing conditions can be chosen to ensure that the final bulk concentration is uniformly distributed and well below the solubility limit.

However, a possible unwanted consequence of implantation is that the primary damage resulting from the ion bombardment may result in secondary defects after annealing. These may contribute to recombination, thus masking the effect of the metal impurities. An effective way to study the impact of any residual secondary damage is to implant a relatively inert species (such as silicon) into silicon, subject the wafers to a typical anneal step, and measure the recombination rate or carrier lifetime as a function of dose.

Indeed it is known that relatively high doses of silicon ions implanted into silicon do result in residual electrically active centers in the near-surface region after high temperature annealing (800-900 $\left.{ }^{\circ} \mathrm{C}\right) .{ }^{1,2}$ However, when implanting metallic ions for lifetime characterization, this near-surface region is etched away after annealing. More recent studies ${ }^{3}$ using carrier lifetime measurements have also shown that high doses of silicon ions result in residual recombination centers, even after annealing at $1000{ }^{\circ} \mathrm{C}$ for $60 \mathrm{~min}$, and that these defects penetrate far below the wafer surface.

The purpose of this work is to identify the threshold dose at which such implantation damage begins to impact on the bulk carrier lifetime after a typical anneal step. This allows safe doses to be chosen for studies of specific metallic impurities. The nature of the recombination centers that cause this residual recombination is also explored in more depth.

\section{EXPERIMENTAL METHODS}

In designing an experiment aimed at characterizing the impact of unidentified recombination centers, an important consideration is their relative recombination strength in either $n$ - or $p$-type silicon. Depending on the energy level and the asymmetry between the capture cross sections for electrons and holes, the recombination activity under low injection of a given defect may be extremely different in these two materials. Hence, in this work, where the capture cross sections and energy levels of the residual defects are not known a priori, we have analyzed the impact of selfimplantation in both $n$ - and $p$-type wafers.

Float zone (FZ) phosphorus-doped $n$-type wafers $(1 \Omega$ $\mathrm{cm})$ and both FZ $(4 \Omega \mathrm{cm})$ and Czochralski-grown $(\mathrm{Cz}, 1 \Omega$ $\mathrm{cm}$ ) boron-doped $p$-type wafers were used. All samples were of $\langle 100\rangle$ orientation. Prior to implantation, the wafers were chemically etched and cleaned. $\mathrm{Si}^{28}$ ions were implanted with an energy of $36 \mathrm{keV}$ in the samples held at $(100 \pm 7){ }^{\circ} \mathrm{C}$ 
and tilted $7^{\circ}$ off axis to minimize ion channeling. Doses between $1 \times 10^{11}$ and $3 \times 10^{16} \mathrm{~cm}^{-2}$ were used, requiring implantation times from several seconds to several hours. After implantation, the wafers were cleaned and annealed in nitrogen gas at $900{ }^{\circ} \mathrm{C}$ for $60 \mathrm{~min}$. This mimics the annealing conditions typically used for distributing small concentrations of implanted metal species in silicon wafers. ${ }^{4}$ Control wafers of each resistivity and type with no implants were coannealed with the implanted wafers and subject to exactly the same cleaning and etching steps. These controls provide a way of determining the recombination rate due to all processes other than those caused by the implant, such as recombination at the surfaces, and recombination in the bulk due to Auger recombination and potential contamination arising from the annealing, etching, and cleaning steps.

After annealing, it is likely that, at least for the higher doses, end-of-range dislocation loops will have formed at the interface of the implanted region and the bulk wafer, as discussed below. These are regions of high recombination, and tend to dominate the measured lifetime. Since metalimplanted samples for lifetime characterization are usually given a shallow etch after annealing to remove any surface precipitation, only the residual recombination centers which penetrate deep into the wafer are of interest here. Consequently, the samples were etched lightly, removing $\approx 5 \mu \mathrm{m}$ per side (much deeper than the peak implant dose which is $\approx 50 \mathrm{~nm}$ ).

After another chemical cleaning step, the samples were subjected to plasma-enhanced chemical-vapor deposition of 70-90 nm thick silicon nitride layers on each surface. This provides surface passivation to allow carrier lifetime measurements using the quasi-steady-state photoconductance method. ${ }^{5}$ With this technique it is possible to measure the lifetime as a function of excess carrier density $\Delta n$, therefore allowing lifetime comparisons between wafers at the same value of $\Delta n$, which, depending on the magnitude of the lifetime, may not correspond to the same illumination intensity. The measured quantity is known as an effective lifetime $\tau_{\text {eff }}$ and incorporates the impact of recombination both in the bulk and at the surfaces.

Deep level transient spectroscopy (DLTS) was performed on the control and selected FZ samples using a modified lock-in-type apparatus. ${ }^{6}$ Schottky barrier diodes were fabricated by thermal evaporation of $\sim 120 \mathrm{~nm}$ of $\mathrm{Au}$ and $\sim 75 \mathrm{~nm}$ of $\mathrm{Ti}$ on the $n$ - and $p$-type samples, respectively. The diameter of the diodes was $1 \mathrm{~mm}$.

\section{DEFECT EVOLUTION DURING ANNEALING}

Much is known about the evolution of implantationrelated defects in self-implanted silicon upon high temperature annealing. ${ }^{7,8}$ The extent of the primary damage in an as-implanted sample depends strongly on the ion energy and the sample temperature during implantation. Higher temperatures result in less damage due to dynamic annealing, which is significant even near room temperature. ' During subsequent high temperature annealing, however, many of the sili- con interstitials and vacancies which constitute this primary damage very quickly recombine, either with each other, or at the surfaces or internal defects.

After this rapid annihilation, the predominant remaining defects are excess interstitials. These can arise from the added silicon atoms themselves, and also interstitials that remain from the primary damage, whose vacancy partners were annihilated at the surfaces. These interstitials tend to form $\{113\}$-oriented defects during annealing at moderate temperatures. ${ }^{7}$ However, these defects are unstable at temperatures above $700{ }^{\circ} \mathrm{C}$. If the implant dose is below a certain critical value, they rapidly dissolve upon further annealing, leaving no trace of secondary defects detectable by DLTS after 15 min at $900{ }^{\circ} \mathrm{C} .{ }^{10}$ However, if the implant dose is higher than the critical value, these defects evolve into end-of-range dislocation loops. ${ }^{7,8}$ These are considerably more stable than the $\{113\}$-oriented defects, and can persist after annealing at temperatures of up to $1000{ }^{\circ} \mathrm{C}$. 3,7

However, at temperatures above $900{ }^{\circ} \mathrm{C}$, these loops also slowly dissolve, injecting silicon interstitials into the wafer bulk. Since, in this work, the near-surface loops themselves are etched away before lifetime measurement, it is the presence of these injected interstitials deep in the wafers that is expected to reduce the carrier lifetime. Other defects, and their dissolution products, are unlikely to be stable enough to still be present under the anneal conditions used.

The critical dose at which secondary defects (dislocation loops) persist after annealing at $900{ }^{\circ} \mathrm{C}$ has been explored previously under various implantation conditions. Jones, Prussin, and Weber found a critical value in the range 1-2 $\times 10^{14} \mathrm{~cm}^{-2}$ for $100 \mathrm{keV} \mathrm{Al} \mathrm{implants,} \mathrm{and} \mathrm{also} \mathrm{for} 50$ and $190 \mathrm{keV}$ B implants, with annealing conditions of $900{ }^{\circ} \mathrm{C}$ for 30 min. ${ }^{7}$ They concluded that, at least for energies below 200 $\mathrm{keV}$, the quantity of added atoms is the determining factor in secondary defect formation, rather than the extent of primary damage. Hence, the critical dose is effectively independent of the implantation energy for low energies, and presumably also of the wafer temperature during implantation. These conclusions were corroborated by the results of Elliman and Mitchell, ${ }^{9}$ who found that the temperature of the wafer during implantation did not play a major role in determining the extent of secondary defects for $540 \mathrm{keV} \mathrm{Si}$ implants annealed at $900{ }^{\circ} \mathrm{C}$ for $15 \mathrm{~min}$.

For higher energy implants in the $\mathrm{MeV}$ range, however, the increased extent of primary damage also impacts on the formation of secondary defects. Schreutelkamp et al. ${ }^{8}$ found a critical dose of $5 \times 10^{13}-1 \times 10^{14} \mathrm{~cm}^{-2}$ for $150 \mathrm{keV} \mathrm{Si} \mathrm{im-}$ plants after annealing at $900{ }^{\circ} \mathrm{C}$ for $15 \mathrm{~min}$, in reasonable agreement with that of Jones, Prussin, and Weber. However, they determined a lower value of $2-4 \times 10^{13}$ for $1 \mathrm{MeV}$ implants. This was confirmed by Kringhøj et al. ${ }^{2,10}$ who also found a value of between $3-6 \times 10^{13} \mathrm{~cm}^{-2}$ for $1 \mathrm{MeV} \mathrm{Si}$ implants annealed at $900{ }^{\circ} \mathrm{C}$ for $15 \mathrm{~min}$ and $1-3$ $\times 10^{13} \mathrm{~cm}^{-2}$ for $5.6 \mathrm{MeV}$ implants. These results led Schreutelkamp to propose that it is the total number of displaced ions which determine the critical dose for a given energy or species. Clearly in the case of low-energy Si implants, a large fraction of these displaced ions may be the added $\mathrm{Si}$ ions themselves. Indeed, simulations by Elliman and 


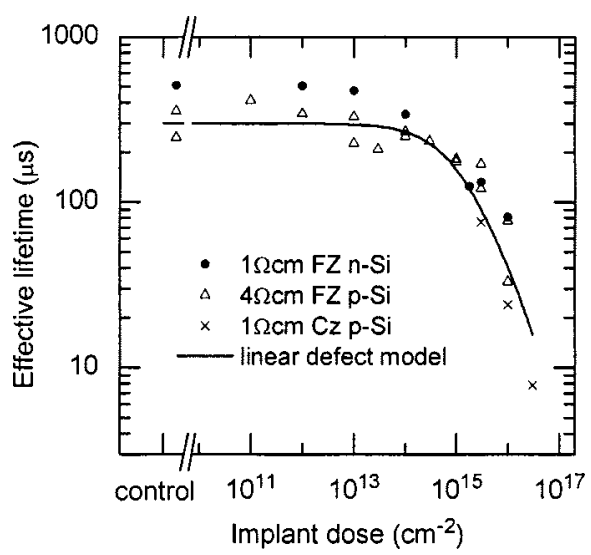

FIG. 1. Effective lifetime measured at $\Delta n=10^{15} \mathrm{~cm}^{-3}$ as a function of $\mathrm{Si}$ implant dose for $n$ - and $p$-type wafers, after annealing at $900{ }^{\circ} \mathrm{C}$ for $60 \mathrm{~min}$ and etching $5 \mu \mathrm{m}$ from each surface. The solid line represents the linear defect model.

Mitchell $^{9}$ showed that for $540 \mathrm{keV} \mathrm{Si}$ implants, the implanted interstitial ions outnumbered the displaced $\mathrm{Si}$ atoms by approximately 5 to 1 before annealing.

The important conclusion from these results is that, for the low-energy implants used here $(36 \mathrm{keV})$, the critical dose for secondary defect formation should be in the range of 5 $\times 10^{13}-2 \times 10^{14} \mathrm{~cm}^{-2}$. In addition, this critical dose should be almost independent of implantation temperature and variations in energy up to $\approx 500 \mathrm{keV}$, at least for light species.

\section{RESULTS AND DISCUSSION}

Figure 1 shows the measured effective lifetimes at an excess carrier density of $\Delta n=1 \times 10^{15} \mathrm{~cm}^{-3}$ as a function of implant dose, after annealing at $900{ }^{\circ} \mathrm{C}$. It is clear that for doses below $10^{13} \mathrm{~cm}^{-2}$, the implanted wafers are, within error, comparable to the control wafers. Hence, there is no additional recombination in these samples, in agreement with DLTS studies on wafers with implants below the critical dose and annealed at $900{ }^{\circ} \mathrm{C} .^{2}$ Note that this threshold value is valid for both the $n$ - and $p$-type wafers, and allows us to conclude that doses below this value are certainly safe to use for preparing metal implanted wafers for lifetime characterization.

However, for doses at and above $1 \times 10^{14} \mathrm{~cm}^{-2}$, the measured carrier lifetime begins to drop. Also shown in Fig. 1 is a linear defect model fitted to the $p$-type data. This simple model assumes that the increase in recombination center density due to secondary defects increases linearly with the implant dose. In this model, the measured lifetime of the control wafers represents the recombination rate due to all processes other than those caused by implantation. The total recombination rate, which is inversely proportional to the measured effective lifetime, is the sum of the rates due to the implanted defects and the "other" channels:

$$
\frac{1}{\tau_{\text {eff }}}=\frac{1}{\tau_{\text {implant }}}+\frac{1}{\tau_{\text {other }}} \text {. }
$$

Figure 1 shows that this model does indeed provide a reasonable fit for the measured data. The fit is also reason-

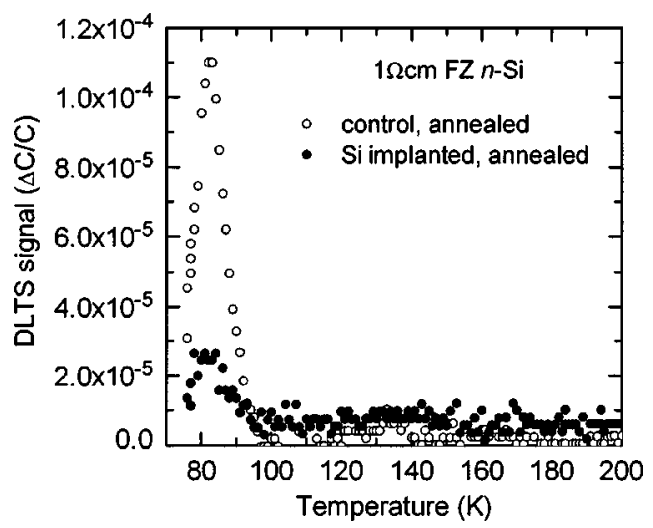

FIG. 2. DLTS spectra for two $n$-type samples: an unimplanted control wafer, and a Si implanted wafer with a dose of $10^{15} \mathrm{~cm}^{-2}$. Both samples were annealed at $900{ }^{\circ} \mathrm{C}$ for $60 \mathrm{~min}$.

able for the $n$-type data after adjusting only the value of $\tau_{\text {other }}$, indicating that the recombination centers introduced have a similar impact on $p$ - and $n$-type silicon.

The threshold dose found here for increased recombination corresponds very well with the critical dose discussed above of $5 \times 10^{13}-2 \times 10^{14} \mathrm{~cm}^{-2}$ for dislocation loop formation. It seems likely then that, in our samples, the slowly dissolving dislocation loops are responsible for this increased recombination.

From the perspective of determining safe doses for metal implants, it is important to consider the origin of the silicon interstitials which form the loops. It may be that many of them are simply those $\mathrm{Si}$ atoms added by the implantation process, as discussed above. In this case, they simply replace the metallic ions normally implanted, and thus cause an underestimation of the maximum safe dose. It is only the recombination due to other interstitials propagated from the implanted region into the bulk that will restrict the safe dose range. However, it is difficult to distinguish between implanted and displaced interstitials, so it suffices to say that the threshold dose determined here is a conservative lower limit.

\section{A. Presence of silicon interstitials}

The presence of silicon interstitials can be detected indirectly with the aid of DLTS. Figure 2 shows DLTS spectra for two $n$-type samples: the control and the sample implanted with $10^{15} \mathrm{~cm}^{-2} \mathrm{Si}$ ions. Recall that these samples were etched after annealing, and so the DLTS spectra probe trapping centers well below the original implanted region. The peak at $83 \mathrm{~K}$ is identified as the vacancy-oxygen center, ${ }^{11}$ and is a measure of the vacancy concentration. Despite the relatively low oxygen content of float-zone silicon (around $10^{15}-10^{16} \mathrm{~cm}^{-3}$ ) compared to Czochralski-grown silicon, there is still ample oxygen for this defect to form in detectable concentrations, as has previously been observed. ${ }^{12}$ From the peak height in Fig. 2, the vacancy-oxygen density is estimated to be in the order of $10^{12} \mathrm{~cm}^{-3}$ in the control sample. Upon implanting and annealing, this peak decreases in magnitude significantly. This reduction in vacancy concentration can be attributed to an increase in the silicon interstitial concentration, arising from the dissolving dislocation loops, 
which annihilate vacancies during annealing. Clearly these vacancy-oxygen complexes cannot be the source of the increased recombination in the implanted samples, since their concentration decreases with dose. However, they do indicate that the silicon interstitial concentration is increased in the implanted samples, as is expected from the creation of dislocation loops for the higher doses. These excess silicon interstitials may form some other complexes, for example, with oxygen or carbon, which result in greater recombination, resulting in the decrease in lifetime seen in Fig. 1.

Majority carrier DLTS spectra on the $n$-type samples only reveal levels in the upper half of the band gap that effectively trap majority carriers or electrons. Corresponding spectra on $p$-type samples up to $280 \mathrm{~K}$ revealed no discernible majority carrier (hole) traps in the lower half of the band gap for an implant dose of $10^{15} \mathrm{~cm}^{-2}$, or in the control sample. Hence, if majority carrier traps due to the excess silicon interstitials are present in these samples, they appear to be below the sensitivity limit of DLTS. Their chemical make up therefore remains unclear.

Note, however, that Fig. 1 also shows results for B-doped $\mathrm{Cz}$ wafers. These wafers contain at least one order of magnitude more interstitial oxygen than the FZ samples, and yet they yield similar lifetimes to the FZ wafers as a function of dose. This tends to indicate that interstitial oxygen is not a key component of the recombination centers generated during annealing.

\section{B. Presence of interstitial iron}

Although the identity of the interstitial-related recombination centers is not clear from the DLTS spectra, it turns out that there is another source of recombination related to the implantation that can be identified. Interstitial $\mathrm{Fe}\left(\mathrm{Fe}_{i}\right)$ in $p$-type silicon forms pairs with the dopant boron atoms ( $\mathrm{FeB}$ pairs). There exists a reliable technique for identifying and determining the concentration of $\mathrm{Fe}_{i}$ by measuring the lifetime before and after breaking the $\mathrm{FeB}$ pairs with strong illumination or light soaking. ${ }^{13,14}$ If $\mathrm{Fe}_{i}$ exists, the lifetime increases after pair breaking at excess carrier concentrations above $\approx 10^{14} \mathrm{~cm}^{-3}$, and decreases below this point. This results in lifetime curves that crossover at this characteristic point, allowing unambiguous identification of $\mathrm{Fe}_{i}$ in borondoped wafers. Also, by measuring the change in lifetime at a given excess carrier density, the concentration of Fe can be determined from the difference in inverse lifetimes in conjunction with a calibration constant determined by the doping level and the carrier density measured. ${ }^{14}$ For the $4 \Omega \mathrm{cm}$ B-doped wafers used here, at an injection level of 1 $\times 10^{15} \mathrm{~cm}^{-3}$, this constant is $-7.2 \times 10^{13} \mu \mathrm{s} \mathrm{cm}^{-3}$.

Figure 3 shows injection-dependent lifetime curves before and after light soaking for $1 \mathrm{~min}$ with white light of approximately one sun intensity $\left(100 \mathrm{~mW} \mathrm{~cm}^{-2}\right)$. Two samples are shown, one which was implanted with $10^{16} \mathrm{~cm}^{-2}$ of $\mathrm{Si}$ ions and another that was not implanted, but contaminated with $\mathrm{Fe}$ by high temperature annealing $\left(1000{ }^{\circ} \mathrm{C}\right)$ in an unclean furnace. Both samples reveal an increase in lifetime at high carrier concentrations, and a crossover point near $10^{14} \mathrm{~cm}^{-3}$. This strongly suggests the presence of $\mathrm{Fe}_{i}$ in both

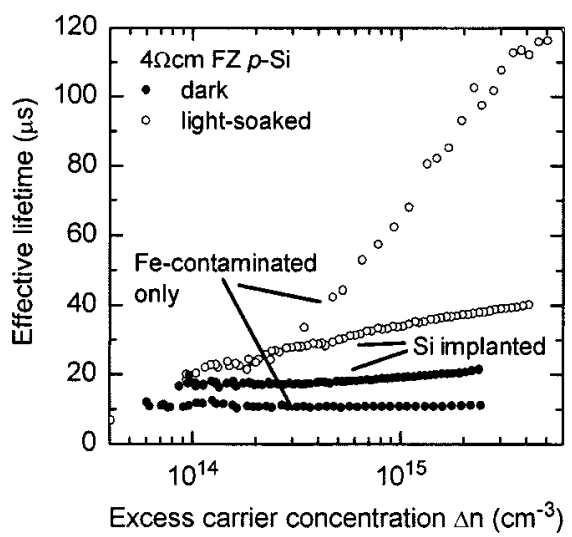

FIG. 3. Injection-dependent lifetime measurements for a $\mathrm{Si}$ implanted $p$-type sample $\left(10^{16} \mathrm{~cm}^{-2}\right.$ dose $)$ and an unimplanted but Fe-contaminated $p$-type sample. Curves are shown before and after light soaking for each sample.

samples. Interstitial Fe produces a center at $0.38 \mathrm{eV}$ above the valence band, but is not visible in the $p$-type DLTS spectra because it is paired with the boron ions to form $\mathrm{FeB}$ pairs, which produce a level in the upper half of the band gap. ${ }^{15}$

From the change in lifetime after light soaking, the concentration of interstitial $\mathrm{Fe},\left[\mathrm{Fe}_{i}\right]$ can be determined. Figure 4 shows how $\left[\mathrm{Fe}_{i}\right]$ (in units $\mathrm{cm}^{-3}$ ) increases as a function of the $\mathrm{Si}$ implant dose (units $\mathrm{cm}^{-2}$ ). The straight line represents a linear function with a constant term of $1.5 \times 10^{11} \mathrm{~cm}^{-3}$. The constant term corresponds to $\mathrm{Fe}$ contamination present even without implantation, and may be caused by furnace contamination or the presence of $\mathrm{Fe}$ in the starting material. Nevertheless, for Si doses above $10^{15} \mathrm{~cm}^{-3}$, the Fe concentration increases linearly with the $\mathrm{Si}$ dose, suggesting contamination in the ion beam. From the linear term in the fit in Fig. 4, and knowing the wafer thickness to be $0.026 \mathrm{~cm}$, it seems that $\approx 4 \mathrm{ppm}$ of the ion beam are in fact $\mathrm{Fe}$ ions.

An important question is how much the presence of parasitic Fe affects the measured lifetimes in Fig. 1. It is conceivable in fact that the linear dependence in Fig. 1 arises solely because of the presence of $\mathrm{Fe}_{i}$, and is not attributable to the presence of Si interstitials at all! The change in lifetime after light soaking for the silicon implanted sample gives an $\mathrm{Fe}_{i}$ concentration of $1.25 \times 10^{12} \mathrm{~cm}^{-3}$, while the $\mathrm{Fe}$ -

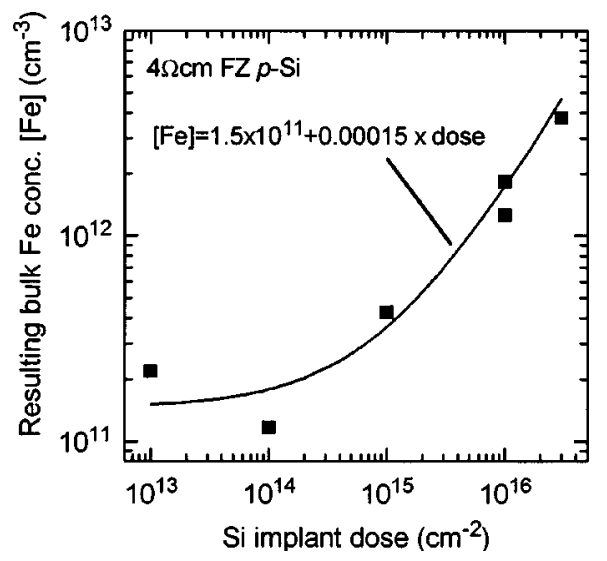

FIG. 4. Bulk Fe concentration as a function of Si implant dose. The solid line shows a linear fit to the data. 
contaminated but unimplanted sample yields a value of 5.5 $\times 10^{12} \mathrm{~cm}^{-3}$. From the known energy level and capture cross sections ${ }^{14}$ of $\mathrm{Fe}_{i}$ we may calculate what the lifetime would be at this carrier density if only $\mathrm{Fe}_{i}$ were present. This yields values of 260 and $60 \mu$ s for these two samples, respectively. For the Fe-contaminated sample, the lifetime due to $\mathrm{Fe}_{i}$ alone is very close to the measured lifetime $(65 \mu \mathrm{s})$, revealing that this sample is in fact completely dominated by the presence of $\mathrm{Fe}_{i}$.

This is not the case for the Si-implanted sample. Using an analog of Eq. (1), it is possible to determine the value of the lifetime in this sample due to all other recombination channels except $\mathrm{Fe}_{i}$. This gives a value of $38 \mu \mathrm{s}$. Alternatively stated, if the relatively small amount of $\mathrm{Fe}_{i}$ in the silicon implanted sample were somehow removed, the measured (light-soaked) lifetime would only increase from 33 to $38 \mu \mathrm{s}$. This indicates that the presence of $\mathrm{Fe}_{i}$ is only responsible for a small fraction of the observed reduction in lifetime after implantation and annealing.

Note that this conclusion is only valid for lifetimes measured after light soaking. Accordingly, all of the lifetime measurements in Fig. 1 are recorded after light soaking for 1 min. The important conclusion is that, in this case, the measured lifetimes are dominated by recombination centers other than $\mathrm{Fe}$. These remaining recombination centers may be related to the presence of the excess silicon interstitials.

\section{CONCLUSIONS}

Lifetime measurements of self-ion implanted silicon wafers have revealed that there is negligible additional recombination in the wafer bulk after annealing at $900{ }^{\circ} \mathrm{C}$ for 60 min for implant doses below $10^{13} \mathrm{~cm}^{-2}$. This indicates the range of doses which are safe to use for preparing metalimplanted samples for lifetime characterization. This threshold value corresponds to the critical dose known to cause the formation of relatively stable dislocation loops during high temperature annealing. It seems that the slow dissolution of these loops results in the injection of silicon interstitials into the wafer bulk, as detected by DLTS. In samples with doses above the threshold value, the recombination center density increases linearly with implant dose, in conjunction with the extent of loop formation. A small proportion of this increased recombination is due to parasitic $\mathrm{Fe}$ contamination in the ion beam. The majority, however, appears to be related to the presence of the excess silicon interstitials in the wafer bulk. Since at least some of the excess interstitials are likely to arise from the implanted ions themselves, rather than atoms they displace, the threshold of $10^{13} \mathrm{~cm}^{-2}$ is in fact a conservative lower limit on which doses are safe to use for other species.

\section{ACKNOWLEDGMENTS}

The authors gratefully acknowledge the assistance of R. Elliman, H. Tan, and C. Jagadish of the Department of Electronic Materials Engineering, ANU, for access to the ion implanter and PECVD reactor. This work has been supported by the Australian Research Council.

${ }^{1}$ S. Fatima, J. Wong-Leung, J. Fitzgerald, and C. Jagadish, Appl. Phys. Lett. 72, 3044 (1998)

${ }^{2}$ P. Kringhøj, S. Fatima, J. S. Williams, and C. Jagadish, Nucl. Instrum. Methods Phys. Res. B 106, 248 (1995).

${ }^{3}$ D. H. Macdonald, H. Maeckel, S. Doshi, W. Brendle, A. Cuevas, J. S. Williams, and M. J. Conway, Appl. Phys. Lett. 82, 2987 (2003).

${ }^{4}$ D. Macdonald, A. Cuevas, and J. Wong-Leung, J. Appl. Phys. 89, 7932 (2001).

${ }^{5}$ R. A. Sinton and A. Cuevas, Appl. Phys. Lett. 69, 2510 (1996).

${ }^{6}$ B. G. Svensson, K.-H. Rydén, and B. M. S. Lewerentz, J. Appl. Phys. 66, 1699 (1989).

${ }^{7}$ K. S. Jones, S. Prussin, and E. R. Weber, Appl. Phys. A: Solids Surf. 45, 1 (1988).

${ }^{8}$ R. J. Schreutelkamp, J. S. Custer, J. R. Liefting, W. X. Lu, and F. W. Saris, Mater. Sci. Rep. 6, 275 (1991).

${ }^{9}$ R. G. Elliman and I. V. Mitchell, Mater. Res. Soc. Symp. Proc. 373, 469 (1995).

${ }^{10}$ P. Kringhøj, J. S. Williams, and C. Jagadish, Appl. Phys. Lett. 65, 2208 (1994).

${ }^{11}$ S. D. Brotherton and P. Bradley, J. Appl. Phys. 53, 5720 (1982).

${ }^{12}$ P. Lévêque et al., J. Appl. Phys. 93, 871 (2003).

${ }^{13}$ G. Zoth and W. Bergholz, J. Appl. Phys. 67, 6764 (1990).

${ }^{14}$ D. Macdonald, L. J. Geerligs, and A. Azzizi, J. Appl. Phys. 95, 1021 (2004).

${ }^{15}$ S. D. Brotherton, P. Bradley, and A. Gill, J. Appl. Phys. 57, 1941 (1985). 